\title{
El trabajo de terminación de especialidad en Medicina General I ntegral y la investigación sobre ambiente- salud
}

\author{
The final paper of Comprehensive General Medicine (Family \\ Medicine) specialty and the environment/ health research.
}

\author{
Jacinta Otero I glesias Lazada';Ethel Bernaza Morales"'Teresa de la Caridad \\ Pérez Díaz'l' \\ 'Máster en Salud Pública y en Educación Médica Superior. Instituto Superior de \\ Ciencias Médicas de La Habana. La Habana, Cuba. \\ "Máster en Medio Ambiente y Desarrollo. Agregada. Instituto de Filosofía del \\ Ministerio de Ciencia, Tecnología y Medio Ambiente. La Habana, Cuba. \\ II'Especialista de II Grado en Medicina General Integral. Profesora Auxiliar. Instituto \\ Superior de Ciencias Médicas de La Habana. La Habana, Cuba.
}

\section{RESUMEN}

IntroducciónLos problemas ambientales no pueden ser exclusivos de los ambientalistas, así en las ciencias de la salud los aspectos relacionados con la integración ambiente y salud son determinantes. La atención primaria de salud es la encargada de consolidar y fortalecer el modelo biopsicosocial y ambientalista. Objetivos Describir las características de la investigación sobre ambiente abordada en los trabajos de terminación de la residencia en la atención primaria de salud. Métodos Se revisaron los informes del trabajo de terminación de la especialidad en Medicina General Integral de la Facultad Calixto García en los años 1990, 1997 y 2006, según las categorías establecidas para el análisis del modelo biopsicosocial y el ambiente.

Resultados Los problemas del ambiente no fueron tratados como objeto de investigación directamente. Los más abordados fueron los estilos de vida, la contaminación del aire, agua y los relacionados con la vivienda. La expresión más frecuente donde subyacen los temas de ambiente en salud fue "factores de riesgo..."

Conclusiones Los componentes o procesos ambientales subyacen en los problemas relacionados con la comunidad, la familia y los individuos, tratados en pocas tesis y no identificados con claridad en las que lo hacen. Se encuentran 
variables del ambiente social, fundamentalmente estilos de vida y problemas psicológicos; del ambiente físico-natural fueron la contaminación del aire y el agua; y del ambiente construido, el problema de la vivienda. Se recomienda preparar a los recursos humanos en salud en la aplicación del enfoque transdisciplinario para el estudio de este problema así como continuar investigando las cuestiones de ambiente en salud encaminadas al fortalecimiento, consolidación e integración del modelo biopsicosocial y ambientalista en el primer nivel de atención.

Palabras claves: Modelo biopsicosocial, ambiente, investigación, trabajo de terminación de especialidad, atención primaria.

\section{ABSTRACTS}

Introduction The environmental problems can not be considered as the environmentalists's exclusive problems because in health sciences, those aspects related to environment and health integration are determining. Primary health care is the one in charge of strengthening the biopsychological and environmentalist model.

Objectives To describe the characteristics of research on environmental issues in the final papers of the primary health care residency students.

Methods The reports from the final papers of Comprehensive General Medicine (Family Medicine) students in "Calixto García" medical school in the years 1990, 1997 and 2006 were reviewed according to the set categories for the analysis of the biopsychological model and the environment.

Results Environmental problems were not directly addressed as a research topic. The most discussed issues in this context were lifestyles, air and water pollution and housing. The most common expression where health environment topics underlie was "risk factors".

Conclusions Underlying environmental components or processes are found in problems related to the community, the family and the individuals, but they were dealt with in a small number of final papers and sometimes unclearly defined. There were variables from the social environment, mainly lifestyles and psychological problems, from the physical/natural environment such as air and water pollution, and from the constructed environment, for example the housing problems. It is recommended to prepare the healthcare human resources for using a transdisciplinary approach to the study of environmental problems and to keep on researching the health environment questions in order to strengthen, consolidate and integrate the biopsychosocial and environmentalist model at the primary healthcare level.

Key words: Biopsychosocial model, environment, research, final paper of residency, primary health care.

\section{NTRODUCCI ÓN}

Históricamente, los temas relacionados con la naturaleza y los problemas sociales han constituido campos del conocimiento diferentes, con esquemas teóricos, 
metodologías y formas de interpretación propias.

Las «ciencias de la naturaleza» y las «ciencias del hombre» dirigían sus esfuerzos a comprender problemas que se cerraban sobre sí mismos. Los problemas de la naturaleza y el ambiente no eran vistos como indispensables para explicar el por qué y el cómo de un determinado orden social. En las últimas décadas, la cuestión ambiental ha cobrado un creciente interés; si en el pasado el aspecto principal para comprender la organización social se encontraba en las relaciones (o en las contradicciones) sociales, el surgimiento de serios inconvenientes ocasionados por el accionar humano sobre el ambiente, ha hecho que contemporáneamente el interés en la relación sociedad-naturaleza se incorpore centralmente a esta interpretación. La preocupación actual por los problemas relacionados con las alteraciones climáticas, la desaparición de la biodiversidad, la acumulación de enormes cantidades de desperdicios industriales y domésticos, la influencia de todo ello en la salud de la población, la participación y responsabilidad del individuo y su comunidad con su salud y el cuidado del ambiente, son ejemplos de ello. ${ }^{1}$

Los problemas ambientales, son por naturaleza, un tema de carácter eminentemente social. Se ha descrito que la determinación social del proceso salud-enfermedad, la necesidad de las ciencias sociales para su comprensión científica y la consiguiente inclusión de estas en el currículo de los centros educacionales donde se forman los profesionales de la salud, son propuestas suficientemente avaladas en el plano científico. Sin embargo, paradójicamente no son de universal aceptación por la comunidad profesional, independientemente de la esfera donde sus miembros desenvuelvan su actividad: docencia, investigación, atención, o administración de salud. Sería más ajustado a la realidad plantear que lo social "es subvalorado", en oportunidades calificado de "no científico" y junto a ello también son subvaloradas determinadas concepciones, políticas y acciones de salud que se sustentan en un enfoque social, no obstante la significativa contribución que pueden brindar a la solución de importantes problemas de salud. ${ }^{2}$ La afirmación realizada en el Programa de Desarrollo de Recursos Humanos de la Organización Panamericana de la Salud (OPS) dice: «la enseñanza de los aspectos preventivos, sociales, colectivos o públicos de la salud en el nivel de pregrado de las profesiones de la salud, no ha recibido atención sistemática en la Región, por lo menos en los últimos 20 años». ${ }^{3}$ Esta aseveración hace suponer que los problemas ambientales siguen el mismo comportamiento dentro del proceso saludenfermedad y el conjunto de actividades relacionadas con ella.

La Salud Pública cubana, ha obtenido profundos logros, basados en un sistema nacional único y gratuito, que se estructura a partir del Programa del Médico y Enfermera de la Familia en una estrecha relación docente-asistencial e investigativa, que se desarrolla mediante la educación en el trabajo y va satisfaciendo las necesidades crecientes de la población. ${ }^{4}$

La especialidad de Medicina General Integral (MGI) constituye el eje del perfeccionamiento del enfoque social de la medicina, que es la premisa básica del Sistema Nacional de Salud. En el programa que sustenta el desarrollo de este tipo de atención se declaran un conjunto de objetivos donde se hace explícito, en general, la importancia del ambiente en el proceso salud-enfermedad. ${ }^{5}$ La temática ambiental está presente, unas veces, declarada explícitamente, por ejemplo: . .... el desarrollo de habilidades para el análisis y solución de problemas ambientales y ecológicos ... " ; y otras donde subyace como es el caso "del desarrollo de habilidades para prevenir enfermedades y lograr cambios en los estilos de vida, resolviendo problemas no determinados dentro del contexto de la atención continuada a sus pacientes y familiares, lo cual sería imposible cumplir con calidad sin tener en cuenta los problemas ambientales." En resumen, las cuestiones relacionadas con ambiente constituyen un eje que transversaliza el Programa por lo que el desarrollo de investigaciones referidas a estas cuestiones relacionandolas 
con su área de atención y acorde con los avances científico-técnicos, debe constituir un reflejo del enfoque de tales problemas.

La investigación científica, a este nivel, en su doble condición de proceso (función de investigación en la formación del especialista de MGI) y resultado (resultados de la investigación que resuelven parcial o totalmente un problema) debe favorecer el desarrollo e integración entre la universidad, los servicios de salud y la sociedad, y aportar información válida que permita conocer el comportamiento de los cambios en el quehacer profesional del personal que se forma en estos escenarios, que tributen hacia un proceder acorde a los principios que rigen el modelo biopsicosocial como eje de la atención de salud en lo cual lo biológico, lo psicológico y lo social forman parte del problema ambiental.

Es por ello que los resultados del trabajo de terminación de la especialización (TTE)*, deben encaminarse, entre otros, a la solución de los problemas planteados en los programas priorizados del MINSAP y estar en correspondencia con el paradigma biopsicosocial que rige este nivel de atención. El TTE debe formar parte de proyectos de investigaciones que se desarrollan en la atención primaria de salud (APS) y donde participan además de los residentes, profesores, tutores y otros especialistas, por lo que el análisis de sus resultados facilita un acercamiento a los problemas que rige la investigación en este nivel y evalua de alguna manera, el desarrollo de aspectos relacionados con el modelo biopsicosocial en general y el tratamiento de los afines al ambiente en particular.

Contar con información científicamente argumentada acerca del abordaje de los problemas del ambiente en los TTE de MGl; conocer cómo y en qué forma son tratados dichos problemas y a su vez que los resultados constituyan indicadores que reflejen, al menos parcialmente, el estado del ambiente en el contexto de la APS, son aspectos imprescindibles a tener en cuenta en cualquier intento de evaluación y control en este nivel de atención para su perfeccionamiento.

El propósito fundamental de este artículo es mostrar aquellos resultados que aportan elementos sobre la incorporación y el tratamiento de los problemas ambientales en los TTE, que pueden contribuir al progreso y fortalecimiento de la investigación en la APS, en correspondencia con la reafirmación de sus principios: promoción, prevención, diagnóstico y tratamiento, rehabilitación y el cuidado del ambiente sustentando el modelo biopsicosocial.

\section{MÉTODOS}

Se realizó una investigación descriptiva de corte transversal en la Facultad "Calixto García", perteneciente al Instituto Superior de Ciencias Médicas de La Habana, durante el período comprendido entre diciembre de 2006 a Febrero de 2007.

El universo de estudio estuvo constituido por todos los TTE de MGI evaluados en los períodos 1990** (66), 1997 (170) y 2006 (82) para un total de 318.

Se seleccionó una muestra no probabilística cuyo tamaño estuvo determinado por la presencia del Informe del TTE en la biblioteca de la facultad (164 TTE para un $51,5 \%$ del universo), la cual quedó compuesta por:

- 38 TTE del año 1990 (23.17\%). 
- 65 TTE del año 1997 (39.63\%).

- 61 TTE del año 2006 (37.20\%).

Los años seleccionados obedecen a criterios de expertos*** y se relacionan con cambios y modificaciones tanto en la APS como en la formación del especialista.

Para cumplimentar los propósitos señalados, se crearon un conjunto de categorías en relación a la ubicación del problema de investigación que se aborda en la tesis, entre las que se encuentran:

- Problemas relacionados con la salud del individuo.

- Problemas relacionados con la salud de la familia.

- Problemas relacionados con la salud de la comunidad.

- Problemas relacionados con el ambiente.

Para particularizar en el tratamiento del ambiente en la investigación, se procedió además a buscar el comportamiento de las siguientes variables o constructos: ambiente físico natural, construido y social, analizando cada uno de ellos en correspondencia con sus componentes.

Otro eje de análisis lo constituyó la acción de salud reflejada en el informe atendiendo a:

- Acciones de promoción.

- Acciones de prevención.

- Acciones de diagnóstico y tratamiento.

- Acciones de rehabilitación.

Se identificaron, además, un conjunto de expresiones redactadas en los informes en los cuales subyace algún contenido relacionado con el ambiente en salud.

En el análisis del contenido de cada TTE se utilizaron los métodos inductivodeductivo, comparativo y el analítico sintético. El resumen del análisis del contenido se recogió en un instrumento diseñado para tales efectos y se creó una base de datos en Microsoft Excel para su procesamiento. Los resultados se presentan en cuadros y gráficos fundamentalmente.

\section{RESULTADOS}

En la tabla 1 se aprecia que el 34,1\% ubica el problema de investigción en la comunidad, seguido del individuo con el 33,5\%. La familia, a pesar de ser, uno de los aspectos más importantes del paradigma biopsicosocial solamente muestra el $8,5 \%$; mientras que los relacionados con el ambiente no se registran. 
El resto de la tabla muestra resultados alentadores en relación con el incremento de los problemas de la comunidad y el individuo, con un aumento de los primeros a expensas de una disminución de los segundos, o sea, 10,5 \%, 18,5\%,65,6\% para la comunidad, mientras que para el individuo fue de $65,8 \%, 38,5 \%$ y $8,2 \%$.

No obstante lo anterior, en la tabla 2 se observa que 67 TTE $(40,8 \%)$ abordaron de alguna forma aspectos relacionados con el ambiente y la mayor frecuencia estuvo en el acápite de "otros", seguido de la comunidad y el individuo con 34,3\%, 28,4 $\%$ y $23,9 \%$, respectivamente.

El ambiente en general, se clasifica en ambiente físico natural, construido y social. En la tabla 3 se ve que el $97 \%$ de las TTE abordaron problemas del ambiente social, el $35,8 \%$ con el ambiente construido, fundamentalmente la vivienda y el físico natural con el $31,3 \%$, que trata aspectos relacionados conla contaminación ambiental del aire en el caso del asma bronquial y de las enfermedades respiratorias con componente alérgico.

La mayoría de los problemas tratados (tabla 4) estuvieron relacionados con problemas de contaminación del aire $(95,2 \%)$ y el agua con el $61,9 \%$ asociados fundamentalmente con enfermedades respiratorias agudas y crónicas para el primer caso y con enfermedades diarreicas agudas en el segundo. El resto de los aspectos contemplados en la tabla no se relacionaban directamente con una enfermedad concreta sino que su tratamiento fue muy genérico dentro de los distintos acápites del informe de investigación.

En relación con el ambiente construido (tabla 5), fue abordado de alguna manera en 22 investigaciones y de ellas el $100 \%$ abordó el componente de la vivienda y $27,3 \%$ el consultorio con un comportamiento similar en ambos años, aunque el problema del consultorio fue mayor en el 2006. No se consignó en ningún trabajo el componente espacios públicos.

En la tabla 6 , se ve que $78,5 \%$ se relacionó con los estilos de vida, seguido de los aspectos psicológicos $(52,3 \%)$, culturales $(32,3 \%)$ y económicos $(30,8 \%)$.

La tabla 7, resume la ubicación del problema de investigación y las formas de ambiente. El ambiente social fue más tratado en los individuos y la comunidad, al igual que el resto de las otras formas de ambiente pero en menor cuantía.

En la tabla 8, se observa, en general, que el diagnóstico y el tratamiento acapararon los problemas en este período estudiado con $50 \%$ de las investigaciones revisadas, seguida de la prevención de salud y la promoción con $22,6 \%$ y $19,5 \%$, respectivamente. Llama la atención como en 1997 disminuyeron las investigaciones relacionadas con el diagnóstico y el tratamiento a expensas de un aumento en las investigaciones relacionadas con la prevención de salud, y la rehabilitación, sin embargo en el año 2006 el aumento de las primeras es ostensible, pues de $38,5 \%$ pasan a $67,2 \%$ en detrimento además del resto de las acciones.

En la tabla 9 se muestra que la expresión más frecuente fue "Factores de riesgo..." con el $15,9 \%$, seguido de "factores predisponentes..." con el 2,4 \%. La primera expresión se ubicó fundamentalmente en problemas relacionados con la comunidad, la familia y el individuo. 


\section{DISCUSIÓN}

Los TTE de MGI, en última instancia, son el reflejo de la actividad investigativa en el nivel primario de atención y a su vez, es una vía para incrementar el conocimiento que contribuya a la solución parcial o total de los problemas sociomédicos y del ambiente que conforman la esencia del paradigma biopsicosocial que debe regir en la APS.

En 1976 un comité de expertos de la Organización Mundial de la Salud (OMS) definió la salud del conjunto de la familia como un hecho que determina y está determinado por la capacidad de funcionamiento efectivo de esta, como unidad biosocial en el contexto de una cultura y sociedad dada y desde entonces quedó aclarado que es un concepto que trasciende el estado físico y mental de sus miembros y se refiere al ambiente sano para el desarrollo natural de los que viven en su seno. ${ }^{6,7}$

Al reflexionar acerca de la presencia de los aspectos del ambiente en cada una una de las ubicaciones del problema de investigación, lo que es de alguna manera satisfactorio si se tiene en cuenta que los mismos constituyen un eje que atraviesa cada una de las ubicaciones reflejadas en este estudio, el hecho podría estar relacionado en este caso con los llamados determinantes de la salud los cuales rebasan el marco de la biología e incluyen los aspectos relacionados con el ambiente, el estilo de vida y el sistema de asistencia de salud. ${ }^{8}$

Los resultados relacionados con las formas de ambiente, son un reflejo de la unión indisoluble entre ambiente y salud, si se tiene en cuenta que una de las primeras causas de morbilidad y mortalidad en niños es la enfermedad respiratoria aguda y el asma bronquial así como las enfermedades diarreicas agudas, las cuales se relacionan generalmente con problemas de contaminación en el aire y el agua. ${ }^{9}$ Por otro lado, de todos es conocido los problemas económicos que presenta el país y el retardo en el cumplimiento en la construcción de viviendas, lo cual ha acarreado serias dificultades no sólo constructivas sino de hacinamiento, lo que conlleva a aumentar la probabilidad de enfermar física y psíquicamente, constituyendo un problema permanente en la vigilancia epidemiológica del estado de salud de la población. En relación con el comportamiento del ambiente social en el aspecto psicológico, se ha afirmado que no existe ningún factor social que no produzca efectos sobre los órganos sensoriales y los sistemas orgánicos (Engels Federico citado por Torrado Luis. Algunas consideraciones, factores y circunstancias en la génesis del delito, 1997). Es la psiquis, el eslabón que une los dos polos, biológico y social, pues satisface las necesidades vitales traduciendo la personalidad en un estilo de vida, que se desarrolla en un contexto ecológico y en un sistema de relaciones sociales y puede valorarse como beneficiosa o perjudicial para la salud (González U. Un enfoque psicológico integral sobre el asma bronquial. TTE, 1997).

En general, los aspectos relacionados con el ambiente social se reflejaron más en el año 1997 que en el 2006 sobre todo aquellos asociados a los estilos de vida y los aspectos psicológicos. Este resultado parece algo contradictorio, ya que debe haber una correspondencia en que a medida que pasa el tiempo se consolide más el modelo biopsicosocial. Los cambios positivos en estilos de vida, son principios fundamentales del quehacer del médico y la enfermera de la familia para lograr el bienestar físico, psíquico y social de su población.

Los problemas de salud vinculados a determinantes de un estilo de vida, pueden ser específicos de una fase de la vida, estar muy relacionados a aspectos culturales, a factores socioeconómicos y otros, que constituyen motivo importante de variaciones en la salud. El tabaquismo, por ejemplo, el cual se eleva a pesar de los 
esfuerzos que se realizan es el primer elemento en relación con el cáncer principalmente de pulmón, que constituye la segunda causa de muerte en Cuba. EI consumo elevado de alcohol causa un gran número de trastornos físicos y psíquicos, el consumo de drogas ilegales, la falta de salud mental, son aspectos que no deben verse aislados del ambiente y la salud sino integralmente. Los problemas citados imponen acciones de prevención, promoción, diagnóstico y rehabilitación si realmente se quiere mantener el equilibrio necesario entre ambiente y salud. ${ }^{10}$

Un aspecto muy importante en el afianzamiento del modelo biopsicosocial, el cual lleva la impronta del ambiente, es la acción de salud que prima en los problemas que se abordan en la investigación en la APS, pues esto consolida el pensamiento y la actividad científica para abordar los problemas en este nivel de atención.

La misión de la APS y de los médicos de familia en Cuba es alcanzar un adecuado estado de bienestar físico, mental, social en personas, familias y comunidades con una amplia participación de los actores sociales, tanto intrasectoriales como extrasectoriales, a través de acciones de promoción, prevención, diagnóstico temprano, tratamiento y rehabilitación. Esto se logrará en los servicios que sean capaces de garantizar, eficiencia, efectividad y la mayor calidad científico-técnica de las actividades que desarrollen; los resultados alcanzados no corroboran esta misión. ${ }^{11}$ La promoción de salud mostró uno de los peores resultados en el tiempo, sin embargo, su visión holística va mas allá de la búsqueda de la salud como un aspecto integral del desarrollo humano. Ella no se limita a la prevención y control de enfermedades, es el enlace entre la verdad científica y la sabiduría popular sobre la condición humana, y la receta indispensable para extender la búsqueda perenne de la felicidad de los individuos y las sociedades, ${ }^{12}$ proporcionando a la población los medios necesarios para mejorar su salud y adoptar un estilo de vida sano.

Para alcanzar un nivel adecuado de bienestar físico, mental y social, las personas o los grupos de población deben poder identificar y satisfacer sus necesidades básicas y su capacidad de cambiar y adaptarse a su entorno. La promoción de la salud, no es dominio exclusivo del sector salud (Lomov BF. Procesos psíquicos y comunicación. En: Problemas metodológicos de psicología social, 1980: p. 151164), abarca muchos componentes: políticas de salud, medidas ambientales, acción de la comunidad, servicios de salud y desarrollo de las aptitudes personales. Todo lo anterior reafirma el carácter complejo de esta actividad. Los problemas que emanan de ella, generalmente se confunden con la actividad en sí misma o la forma de desarrollarla. Por ejemplo, charlas sanitarias, carteles, spot de televisión y otras, sin embargo y como se observa en estos resultados los problemas no son abordados científicamente. ${ }^{13}$ El tema ambiental no puede ser una cuestión exclusiva de los ambientalistas. Al ser el ambiente aquello donde vivimos, y de lo que vivimos, tenemos necesidad de admitirlo e incluirlo en todas las esferas de nuestra realidad.

Dado que lo ambiental se define como el campo de intersección entre la naturaleza y la sociedad, la comprensión de cualquier problema exige la intervención de las ciencias naturales, tecnológicas y sociales. Ello requiere, por tanto un comportamiento nuevo ante la ciencia, ya que la perspectiva ambiental ha puesto en claro la necesidad de afrontar el análisis de sistemas complejos (como son los sistemas ambientales) desde las perspectivas de las diferentes ciencias. Organizar el trabajo interdisciplinario no consiste sólo en reunir ante una mesa de estudio a profesionales de distintas ramas del saber. Se trata de crear nuevas formas de comprensión de la realidad; exige un cambio de actitud entre los investigadores para asumir nuevos métodos, que pueden ser resultado de la simbiosis de los ya existentes. ${ }^{14}$ La asimilación conciente del problema ambiental por las ciencias de la salud y su penetración cada vez más profunda, posibilita un salto cualitativamente superior en su desarrollo. ${ }^{2}$ 
Se ha visto en los resultados expresados, que los problemas ambientales no son abordados directamente en las investigaciones desarrolladas en la APS; aunque no es menos cierto, que en parte de ellas se tratan estos temas, unas veces directamente relacionados a problemas de salud y otras apoyando algunas de sus fundamentaciones sin que quizás, se tenga una real conciencia de ello por parte de los investigadores.

En las investigaciones en salud los factores de riesgo (o criterios de riesgo) son aquellas características y atributos (variables) que se presentan asociados directamente con la enfermedad o el evento estudiado; ellos no son necesariamente las causas (o la etiología necesaria), sólo sucede que están asociadas con el evento. ${ }^{15,16}$ La noción de riesgo epidemiológico es función de la existencia del ser humano viviendo en un ambiente social en variación permanente, vale decir, los riesgos que afectan la salud, dependen de la vida en sociedad, y más precisamente, del tipo estructural de la sociedad en que se vive. ${ }^{17,18} \mathrm{El}$ hombre, que está en un ambiente sociocultural artificial, creado y desarrollado históricamente por él mismo, tiene por razones ecológicas y sociales una diversidad grande de riesgos y una oportunidad también mayor de enfrentarse a ellos. ${ }^{19}$

Es lógico pensar que los problemas relacionados con el riesgo de enfermar del individuo, la comunidad y la familia sean los problemas fundamentales que se aborden en la investigación en salud, pero como se ha visto falta la concepción de que el ambiente también es susceptible de enfermar y repercutir directamente en la salud de la población o potencializar la acción de otros factores. La consolidación, el impulso y la importancia del modelo ambientalista en su relación con otros paradigmas y en ese caso el modelo biopsicosocial, permitirán el enfoque integral y holístico de los problemas que afectan la salud de las poblaciones.

A modo de resumen y como conclusiones, se pude decir que el ambiente no aparece declarado directamente como objeto de estudio en los trabajos de terminación de la especialidad de Medicina General Integral analizados. En menos de la mitad de los informes analizados, los componentes o procesos ambientales subyacen en los problemas relacionados con la comunidad, la familia y los individuos, pero no se identifican con claridad. En primer lugar, se abordan variables del ambiente social y dentro de este se trata fundamentalmente, estilos de vida y problemas psicológicos. En segundo lugar, se investiga sobre el ambiente físico-natural principalmente, la contaminación del aire y el agua; y por último, el ambiente construido, esencialmente el problema de la vivienda.

La acción de salud que prima en la mitad de las investigaciones revisadas es el diagnóstico y el tratamiento. Acciones tan importantes y relacionadas con los problemas de ambiente en la atención primaria de salud como la promoción y la prevención, han sido poco tratados. Al relacionar la acción de salud con las formas de ambiente fue el ambiente social el más vinculado con estas acciones aunque con muy poca participación, aproximadamente la décima parte de los trabajos de terminación de la especialidad lo abordaron.

De las expresiones identificadas, que pudieran tener relación con el ambiente, los "factores de riesgo" tuvieron el mayor peso asociados a investigaciones sobre la comunidad y la familia principalmente.

Finalmente se pueden sugerir, a modo de recomendaciones, examinar la posible ampliación de los contenidos relacionados con ambiente y salud en los distintos programas de formación de los recursos humanos en salud, en los niveles de pre y posgrado, de forma que se consiga preparar a los estudiantes en la aplicación del enfoque transdisciplinario que se requiere. Continuar investigando sobre los 
problemas de ambiente en salud en la atención primaria, como forma de retroalimentación y para la toma de decisiones encaminadas al fortalecimiento, consolidación e integración del modelo biopsicosocial y ambientalista en este nivel de atención.

\section{REFERENCI AS BI BLI OGRÁFICAS}

1. Guido P. Galafassi. Aproximación a la problemática ambiental desde las ciencias sociales. Un análisis desde la relación naturaleza-cultura y el proceso de trabajo. Theorethikos, año 1, no 6, 1998.

2. Pérez Cárdenas M. Lectura de filosofía, salud y sociedad. Los paradigmas médicos: factores de su conservación y cambio. La Habana: Editorial Ciencias Médicas; 2000.

3. Vela Valdés J. La enseñanza de las disciplinas de Salud Pública en la carrera de Medicina. Situación actual y perspectivas [informe]. La Habana: MINSAP, Instituto de Ciencias Médicas de La Habana; 1994.

4. Ministerio de Salud Pública (Cuba). Organización, desarrollo y control del proceso docente educativo en la Atención primaria de salud (APS). La Habana: MINSAP, área de docencia e investigación; 2002.

5. López J. Las unidades docentes de atención primaria. Rev Cub Med.Fam. 2000; 18 (1): 70-6.

6. Lauro Bernal I. Modelo de salud del grupo familiar. Rev Cubana Salud Pública. 2005; 31(4).

7. Organización Mundial de la Salud. Índices estadísticos de la salud de la familia. Informe de un Comité de Expertos. Ginebra: OMS; 1976. (Serie de Informes Técnicos; No. 587).

8. Ordóñez Gonzalo A. Salud Ambiental: Concepto y actividades. Rev Salud Pública. 7(3) 2000.

9. Ministerio de salud Pública (Cuba). Anuario estadístico de Salud.La Habana: MI NSAP, Dirección nacional de estadísticas; 2004.

10. Determinantes de salud. Salud Pública. Comunidad Europea [serie en Internet]. [citado Abr 2007]. Disponible en: www.google.com

11. Colectivo de Autores. Introducción a la Medicina General Integral. La Habana: Editorial Ciencias Médicas; 2001.

12. Promoción de la salud: una antología. Publicación Científica \# 557. Washington, D.C.: OPS; 1996.

13. Barcaz Hechavarría E. Estrategia de comunicación social para la atención primaria de salud en la provincia Las Tunas. Rev Cubana Educ Med

Super. 2002; 16(11). 
14. Mateo J. La ciencia y la tecnología en el debate ambiental. Filosofía y sociedad. La Habana: Editorial Félix Varela; 2000.

15. Narey R, Aldereguía J. Medicina social y salud pública en Cuba. La Habana: Editorial Pueblo y Educación; 1990.

16. Rodríguez Calzadilla A. Enfoque de riesgo en la atención primaria de estomatología. Rev Cubana Estomatol. 1997; 18 (1):40-9.

17. San Martín H, Martín AC, Carrasco JL. Epidemiología. Investigación práctica. Madrid: Díaz de Santos; 1990.

18. GO, Caplen DJ. Methodological issues in longitudinal epidemiologic dental caries. Community Dent Oral Epidemiol. 1999;2 (4):236-48

19. Duque de Estrada Riverón J, Rodríguez Calzadilla A. Factores de riesgo en la predicción de las principales enfermedades bucales en los niños. Rev Cubana Estomatol. 2001;38(2).

Recibido: 4 de marzo de 2008.

Aprobado: 4 de abril de 2008.

Jacinta Otero Iglesias Lazada. Norte Primera núm.705 entre Avenida Norte y Avenida Sur. Reparto Santa Catalina. Cerro, La Habana.

Teléf.: 6 40-06-59. E-mail: teobaldo.marino@infomed.sld.cu

* Es el informe final escrito de la investigación realizada por el médico residente durante el periodo de la especialización. Debe estar relacionado con los problemas de salud de la comunidad e integrado a las estrategias de investigación en la atención primaria de salud.

** Se toma el año 1990 ya que anterior a esta fecha la formación del especialista se realizó con programas provisionales.

*** Profesores y directivos que participan en el proceso de formación del especialista de MGI desde sus comienzos en el curso académico 1983-1984. anterior a esta fecha la formación del especialista se realizó con programas provisionales.

*** Profesores y directivos que participan en el proceso de formación del especialista de MGI desde sus comienzos en el curso académico 1983-1984. 
Tabla 1. Trabajos de teminación de la especialidad segirn la ubicación del problema de irvestigación y afio. Facultad Calixto Garcia. 1990,1997 y 2016

\begin{tabular}{|l|l|l|l|l|l|l|l|l|}
\hline \multirow{2}{*}{ Ubicación } & \multicolumn{2}{|c|}{1990} & \multicolumn{2}{c|}{1907} & \multicolumn{2}{c|}{2006} & \multicolumn{2}{c|}{ Total } \\
\cline { 2 - 9 } & No. & $\%$ & No. & $\%$ & No. & $\%$ & No. & $\%$ \\
\hline Individuo & 25 & 65,8 & 25 & 38,5 & 5 & 8,2 & 55 & 33,5 \\
\hline Farrilia & 5 & 13,2 & 8 & 12,3 & 1 & 1,6 & 14 & 8,5 \\
\hline Cornuridad & 4 & 10,5 & 12 & 18,5 & 40 & 65,6 & 56 & 34,1 \\
\hline Otros & 4 & 10,5 & 20 & 30,8 & 15 & 24,6 & 39 & 23,8 \\
\hline Total & 38 & 100 & 65 & 100 & 61 & 100 & 164 & 100 \\
\hline
\end{tabular}

Tabla 2. Trabajos de temiración de la especialidad segiru la frecuencia de aspectos relacionados con el arb iente que subyacer en el conterido de los rismos.

Facultad Calixto Garcia. 1997 y 2006

\begin{tabular}{|l|l|l|l|l|l|l|}
\hline \multirow{2}{*}{ Ubioción } & \multicolumn{2}{|c|}{1997} & \multicolumn{2}{c|}{2006} & \multicolumn{2}{c|}{ Total } \\
\cline { 2 - 7 } & 150. & $\%$ & No. & $\%$ & No. & $\%$ \\
\hline Individuo & 13 & 35,1 & 3 & 10 & 16 & 23,9 \\
\hline Farrilia & 8 & 21,6 & 1 & 3,3 & 9 & 13,4 \\
\hline Comuridad & 3 & 8,1 & 16 & 53,3 & 19 & 28,4 \\
\hline Otros & 13 & 35,1 & 10 & 33,3 & 23 & 34,3 \\
\hline Total & 37 & 100 & 30 & 100 & 67 & 100 \\
\hline
\end{tabular}

Tabla 3 . Trabajos de temiración de la especialidad segin formas de abordaje del ambiente. Facultad Calixto Garia. 1997 y 2006

\begin{tabular}{|c|c|c|c|c|c|c|}
\hline \multirow{2}{*}{ Formas } & \multicolumn{2}{|c|}{1997} & \multicolumn{2}{|c|}{2006} & \multicolumn{2}{|c|}{ Total } \\
\hline & No. & $\beta^{+\infty}$ & No. & $\psi^{*+\ldots}$ & No. & 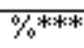 \\
\hline \begin{tabular}{|l} 
Fisico natural \\
\end{tabular} & 15 & 40,5 & 6 & 20,0 & 21 & 31,3 \\
\hline Corstruido & 14 & 37,8 & 8 & 26,7 & 24 & 35,8 \\
\hline Social & 37 & $10 \overline{1}$ & 28 & 93,3 & 65 & 97,0 \\
\hline
\end{tabular}

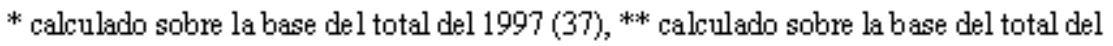
$2006(30)$, ***:* calculado sobre la base del total del general $67 \%$. 
Tabla 4. Frecuencia de los componentes del arbiente fisico natural abordados en los trabajos de terrimación de la especialidad por ario.

Farultad Calixto Garcia. 1997 y 2006

\begin{tabular}{|c|c|c|c|c|c|c|}
\hline \multirow{2}{*}{ Fisico ratural } & \multicolumn{2}{|c|}{1997} & \multicolumn{2}{|c|}{2006} & \multicolumn{2}{|c|}{ Total } \\
\hline & No. & $\% *$ & No. & 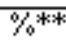 & No. & $\%^{+x+*+*}$ \\
\hline Aire & 14 & 93,3 & 6 & 100 & 20 & 95,2 \\
\hline Flora & 6 & 40 & 4 & 66,6 & 10 & 47,6 \\
\hline Suelo & 6 & 40 & 4 & 66,6 & 10 & 47,6 \\
\hline Famia & 6 & 40 & 4 & 66,6 & 10 & 47,6 \\
\hline Agua & 9 & 60 & 4 & 66,6 & 13 & 61,9 \\
\hline Biológico & 4 & 26,7 & 2 & 33,3 & 6 & 28,6 \\
\hline
\end{tabular}

*aleulado sobre la base del total (15), **alculado sobre la base del total $(6)$, ****alculado sobre la base del total (21)

Tabla 5 . Frecuencia de los componentes del ambiente constnuido abordados en los trabajos de temuinación de la especialidad por afro.

Facultad Calixto Garcia. 1997 y 2006

\begin{tabular}{|c|c|c|c|c|c|c|}
\hline \multirow{2}{*}{ Arubierte corstruido } & \multicolumn{2}{|c|}{1997} & \multicolumn{2}{|c|}{20106} & \multicolumn{2}{|c|}{ Total } \\
\hline & No. & $\% *$ & Wo. & $\%^{+* *+}$ & Wo. & $\%_{0}^{*+* * *+1}$ \\
\hline Tivienda & 14 & 100 & 8 & 100 & 22 & 100 \\
\hline Corsultario & 2 & 14,3 & 4 & 50 & 6 & 27,3 \\
\hline
\end{tabular}

*alculado sobre la base del total (14), ***alculado sobre la base del total (8), ****alculado sobre la base del total (22).

Tabla 6. Frecuencia de los comporentes del armbiente social abordados en los trabajos de termiriación de la especialidad por año.

Facultad Calixto Garcia. 1997 y 2006

\begin{tabular}{|l|l|l|l|l|l|l|}
\hline \multirow{2}{*}{ Armbiente Social } & \multicolumn{2}{|c|}{1997} & \multicolumn{2}{c|}{2006} & \multicolumn{2}{c|}{ Total } \\
\cline { 2 - 7 } & $1 \% 0$. & $\% *$ & No. & $\%$ ** & No. & $\%$ क** \\
\hline Estilos de vida & 31 & 83,8 & 20 & 71,4 & 51 & 78,5 \\
\hline Aspectos ecorómicos. & 11 & 29,7 & 9 & 32,1 & 20 & 30,8 \\
\hline Aspectos culturales & 12 & 32,4 & 9 & 32,1 & 21 & 32,3 \\
\hline Aspectos psicológicos & 23 & 62,2 & 11 & 39,3 & 34 & 52,3 \\
\hline
\end{tabular}

*alculado sobre la base del total (37), ***alculado sobre la base del total (28), ***:*alculado sobre la base del total (65). 
Tabla 7. Trabajos de terminación de la especialidad segirn la ubicación del problema de irwestigación $y$ formas de abordaje del medio arob iente.

Facultad Calixto Garcia. 1997 y 2006

\begin{tabular}{|l|l|l|l|l|l|l|}
\hline \multirow{3}{*}{ Ubicación } & \multicolumn{4}{|l|}{ Formas } \\
\cline { 2 - 7 } & \multicolumn{2}{|c|}{ Físico natural } & \multicolumn{2}{|c|}{ Construido } & \multicolumn{2}{c|}{ Social } \\
\cline { 2 - 8 } & No. & $\%$ & No. & $\%$ & No. & $\%$ \\
\hline Irdiriduo & 8 & 50 & 12 & 75 & 16 & 100 \\
\hline Farrilia & 1 & 11,1 & 2 & 22,2 & 9 & 100 \\
\hline Corrurridad & 5 & 26,3 & 3 & 15,8 & 19 & 100 \\
\hline Otros & 6 & 26,1 & 5 & 21,7 & 21 & 91,3 \\
\hline
\end{tabular}

Porcentaje calculado en base al total de cada ubic ación (total de la tabla 2).

Tabla 8. Trabajos de teminación de la especialidad segin resultados de la acción de salud $y$ afio. Facultad Calixto Garcia. 1990,1997 y 2006

\begin{tabular}{|l|l|l|l|l|l|l|l|l|}
\hline \multirow{2}{*}{ Ección de salud } & \multicolumn{2}{|c|}{1990} & \multicolumn{2}{c|}{1907} & \multicolumn{2}{c|}{2006} & \multicolumn{2}{c|}{ Total } \\
\cline { 2 - 9 } & No. & $\%$ & No. & $\%$ & 150. & $\%$ & No & $\%$ \\
\hline Fromación & 13 & 34,2 & 12 & 18,5 & 7 & 11,5 & 32 & 19,5 \\
\hline Prevernión & 6 & 15,8 & 20 & 30,8 & 11 & 18 & 37 & 22,6 \\
\hline $\begin{array}{l}\text { Diagróstico } \\
\text { y tratamuento }\end{array}$ & 16 & 42,1 & 25 & 38,5 & 41 & 67,2 & 82 & 50 \\
\hline Fehabilitación & 3 & 7,9 & 8 & 12,3 & 2 & 3,3 & 13 & 7,9 \\
\hline Total & 38 & 100 & 65 & 100 & 61 & 100 & 164 & 100 \\
\hline
\end{tabular}

Tabla 9. Expresiones más usadas para el abordaje del amb iente en los trabajos de terrumación de la especialidad se girm ubic ación del problema de irvestigación.

$$
\text { Facultad Calixto Garia. } 1997 \text { y } 2006
$$

\begin{tabular}{|c|c|c|c|c|c|c|c|c|c|c|}
\hline \multirow{3}{*}{ Expresiones } & \multicolumn{8}{|c|}{ Ubicación } & \multirow{2}{*}{\multicolumn{2}{|c|}{ Total }} \\
\hline & \multicolumn{2}{|c|}{ Indriduo } & \multicolumn{2}{|c|}{ farrilia } & \multicolumn{2}{|c|}{ Cormuridad } & \multicolumn{2}{|c|}{ Otros } & & \\
\hline & No. & $\%$ & No. & $\%$ & No. & $\%$ & No. & $\%$ & No. & $\%$ \\
\hline $\begin{array}{l}\text { Factores } \\
\text { de ries go... }\end{array}$ & 8 & 14,5 & 4 & 28,6 & 17 & 30,4 & 7 & 17,9 & 26 & 15,9 \\
\hline $\begin{array}{l}\text { Factores } \\
\text { deteminantes... }\end{array}$ & 2 & 3,6 & 0 & 0 & 0 & 0 & 1 & 2,6 & 3 & 1,8 \\
\hline $\begin{array}{l}\text { Irfluercia } \\
\text { de factores... }\end{array}$ & 0 & 0 & 0 & 0 & 0 & 0 & 1 & 2,6 & 1 & 0,6 \\
\hline $\begin{array}{l}\text { Factores } \\
\text { predipponentes... }\end{array}$ & 2 & 3,6 & 0 & $\overline{0}$ & 2 & 3,6 & 0 & $\overline{0}$ & 4 & 2,4 \\
\hline $\begin{array}{l}\text { Condiciones } \\
\text { de riesgo... }\end{array}$ & 0 & 0 & 0 & 0 & 1 & 1,8 & 0 & 0 & 1 & 0,6 \\
\hline
\end{tabular}

Porcentaje calculado en base al total de cada ubicación (total de la tabla 2). 